\title{
Sleep-wake disturbances in the premotor and early stage of Parkinson's disease
}

\author{
Panagiotis Bargiotas, Michael W.M. Schuepbach, and Claudio L. Bassetti
}

\begin{abstract}
Purpose of review
Review of recent literature pertaining to frequency, associations, mechanisms, and overall significance of sleep-wake disturbances (SWD) in the premotor and early phase of Parkinson's disease.

\section{Recent findings}

SWD are frequent in Parkinson's disease and their prevalence increases with disease progression. Recent studies confirm previous findings that SWD can appear as initial manifestation of Parkinson's disease even decades before motor signs appear and highlight their clinical associations in these early stages. More intriguingly, new evidence underpins their role as risk factors, predictors, or even as driving force for the neurodegenerative process. As our understanding of sleep-wake neurobiology increases, new hypotheses emerge concerning the pathophysiology of SWD in early Parkinson's disease stages involving dopaminergic and nondopaminergic mechanisms.
\end{abstract}

\section{Summary}

SWD are predictors for the development of parkinsonian syndromes including Parkinson's disease. This may offer the opportunity of developing new preventive strategies and interventions at an early stage of this neurodegenerative disease.

\section{Keywords}

biomarkers, daytime sleepiness, insomnia, prodromal Parkinson's disease, REM sleep behavior disorder

\section{INTRODUCTION}

Sleep-wake disturbances (SWD) are frequent in Parkinson's disease and are typically attributed to Parkinson's disease medications, poor sleep hygiene, and other nonmotor symptoms (NMS) such as nocturnal akinesia, pain, cramping, and nycturia. Over the past decade, notable advances have been made in the understanding of the role of the dopaminergic system in circadian mechanisms and sleep-wake physiology [1]. In addition, nondopaminergic systems are involved early in the pathogenesis of SWD in Parkinson's disease [2,3]. These findings suggest that SWD are no longer solely a complication of advanced PD but can emerge, among other NMS, in the premotor stages as a primary manifestation of neurodegeneration [4] and increase in frequency with Parkinson's disease progression (Fig. 1). Considering the importance of early diagnosis and intervention, the possibility that SWD may have prognostic value for the development of Parkinson's disease is intriguing.

This review will discuss first the most recent literature on SWD in the context of premotor and early stage of Parkinson's disease and then important recent evidence on the pathophysiology of SWD in early Parkinson's disease.

\section{SUBJECTIVE SLEEP DISTURBANCES/ INSOMNIA}

Two thirds of patients with Parkinson's disease report poor sleep quality [5], many of them very early in the course of the disease [6,7]. Recent studies confirm the high prevalence of subjective sleep disturbances in the early stages of Parkinson's disease and highlight their association with low quality of life $\left[8,9^{-*}, 10^{-"}\right]$ and other NMS such as global cognitive impairment, anxiety, depression and nocturia [8]. Individuals later diagnosed with

Department of Neurology, University Hospital Bern and University of Bern, Bern, Switzerland

Correspondence to Prof. Dr med. Claudio L. Bassetti, Chairman and Director, Department of Neurology, Inselspital, University Hospital Bern, Freiburgstrasse 18, CH- 3010, Bern, Switzerland. Tel: +41(0)31 63230 66; fax: +41 (0)31 63296 79; e-mail: claudio.bassetti@insel.ch

Curr Opin Neurol 2016, 29:763-772

DOI:10.1097/WCO.0000000000000388 


\section{KEY POINTS}

- Sleep-wake disturbances occur years prior to the onset of motor Parkinson's disease signs, and their frequencies increase with disease progression.

- New data are emerging concerning the pathophysiology of sleep-wake disturbances in early Parkinson's disease, implicating dopaminergic and nondopaminergic mechanisms.

- Premotor sleep-wake disturbances and mainly REM sleep behavior disorder have the potential to serve as prodromal markers of Parkinson's disease. The reliable identification of individuals at high risk of developing Parkinson's disease could become crucial when preventive or disease-modifying therapies become available.

Parkinson's disease had, years before Parkinson's disease diagnosis, a higher incidence of sleep disturbances [10"'] and insomnia [11"] than controls. Baig et al. [9"'] showed that 769 newly diagnosed patients with Parkinson's disease experienced significantly more sleep disturbances than first-degree Parkinson's disease relatives and control subjects. Sleep disturbances emerged in $60 \%$ of untreated and in $70 \%$ of treated patients with
Parkinson's disease, suggesting that either the disease progression or the introduction of dopaminergic medication may negatively impact sleep quality. In the same study, patients with tremor dominant Parkinson's disease showed a better sleep profile than other nontremor-dominant phenotypes [9"'].

In agreement with the reported subjective sleep disturbances, previous polysomnographic studies showed that patients with Parkinson's disease exhibit significant abnormalities in sleep architecture not only in fully developed motor Parkinson's disease [12] but in the early phase of the disease as well. These abnormalities include increased sleep latency, reduced sleep efficiency, reduced rapid eye movement (REM) sleep [7], and significant changes in the REM and non-REM sleep architecture [13-16].

Recently, Margis et al. [17"] examined differences in sleep architecture between newly diagnosed, nondepressed, nondemented, drugnaïve patients with Parkinson's disease $(n=8)$ and age- and sex-matched controls, using scalp electroencephalography (EEG). In patients with Parkinson's disease, alpha and sigma activity during non-REM sleep was significantly higher in almost all examined brain regions [17"]. Considering that sigma activity is associated with sleep stability [18]

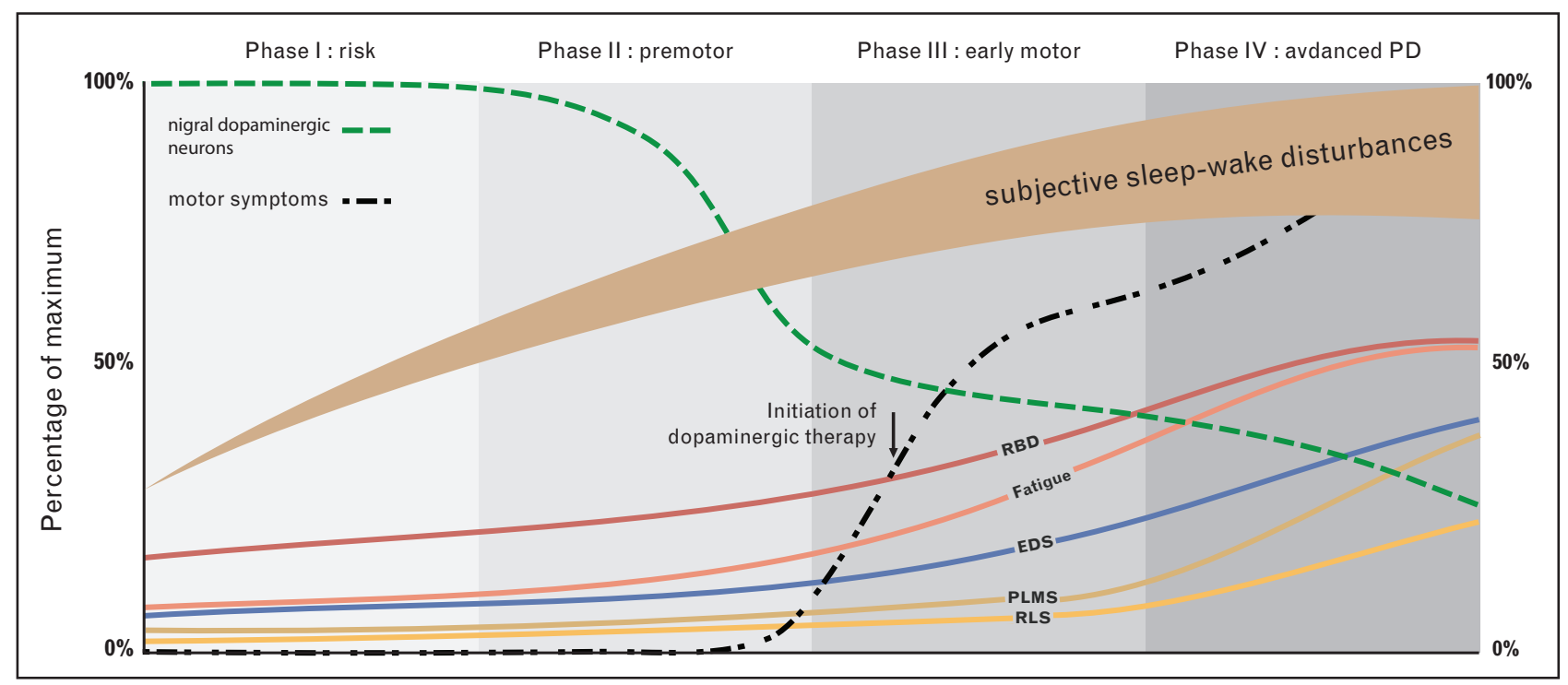

FIGURE 1. Sleep-wake disturbances over the course of Parkinson's disease. Subjective sleep-wake disturbances are prevalent in all stages of Parkinson's disease (phase I-IV) and present in almost all patients with advanced Parkinson's disease. In individuals at high risk of developing Parkinson's disease, RBD symptoms (mainly dream-enacting behavior), fatigue, and probably EDS are more frequently reported than in the general population even a decade prior to onset of motor Parkinson's disease signs (phase I). Gradually, as neuronal degeneration progresses, SWD increase in frequency and their spectrum expands. Dopaminergic medication often provides a relief on motor Parkinson's disease signs but has only a minor (or even a negative) impact on sleep-wake disturbances. EDS, excessive daytime sleepiness; PLMS, periodic limb movements in sleep; RBD, REM sleep behavior disorder; RLS, restless legs syndrome. 
and processing speed [19] and, as an important determinant of spindle production, with cognitive abilities [20,21], these findings contradict previous reports that patients with Parkinson's disease without REM sleep behavior disorder (RBD) showed a significantly lower sleep spindle density in nonREM sleep when compared to matched controls [22]. The inclusion of treated patients with Parkinson's disease in the Danish study may be a reasonable explanation for this discrepancy. However, the impact of dopaminergic medication on sleep architecture was not confirmed in a recent polysomnographic study. In 15 newly diagnosed patients with Parkinson's disease, after initiation of dopaminergic treatment, subjective sleepiness (assessed by Epworth sleepiness scale, ESS) improved but no significant changes in sleep macroarchitecture and other objective sleep parameters were found [23].

\section{DISRUPTION OF CIRCADIAN RHYTHM}

Several normal circadian patterns are desynchronized in Parkinson's disease including diurnal fluctuation of sleep-wake cycles, cardiovascular (blood pressure, heart rate), and sensory functions [24"]. Recent studies focusing on circadian rhythmicity in early Parkinson's disease are limited. Breen et al. [7] showed that circadian alterations are linked to differences in peripheral clock gene expression and to fluctuations on circulating hormones also in early Parkinson's disease. Although there was no evidence of a melatonin phase shift in patients with Parkinson's disease compared to controls, alterations in melatonin secretion were significantly correlated with objective sleep parameters including sleep latency and reduced slow-wave sleep [7]. Interestingly, Lauretti et al. showed that circadian rhythm disruption may contribute to 1-methyl-4-phenyl-1,2,3,6-tetrahydropyridine (MPTP)-induced neurotoxicity, suggesting that circadian disturbances might be a driving force for neurodegenerative processes [25]. However, symptomatic treatment with exogenous melatonin in patients with idiopathic RBD did not affect neurodegenerative outcome [26"']. Therefore, the hypothesis of circadian rhythm disorder as an environmental or endogenous risk factor for developing Parkinson's disease needs further investigation.

\section{RAPID EYE MOVEMENT SLEEP BEHAVIOR DISORDER}

In patients with Parkinson's disease, RBD is prevalent in up to 50\% (varying between 15 and 60\%)
$[27,28]$, worsens with disease progression $[29,30]$, and has a negative impact on quality of life [31].

\section{Rapid eye movement sleep behavior disorder is part of prodromal Parkinson's disease}

In the last decade, many studies provided evidence that RBD precedes Parkinson's disease motor signs by several years, suggesting that RBD should no longer be considered a complication but a part of the prodromal phase of the disease [13,32]. Recent studies confirm this notion: in the Parkinson's Progression Markers Initiative (PPMI) study, possible RBD emerged in $25.5 \%$ of drug-naïve patients with Parkinson's disease [33"]. In 1719 newly diagnosed Parkinson's disease cases, the prevalence of RBD, assessed by RBD questionnaire (RBDQ), was 43\% [34]. RBD in the premotor Parkinson's disease phase is associated with other NMS including neuropsychiatric symptoms, such as hallucinatory phenomena, axial symptoms, and cognitive impairment [33',34,35]. Furthermore, dream enactment was frequently perceived more than 10 years before the onset of Parkinson's disease motor signs [10""] and was reported more frequently by individuals at high risk for Parkinson's disease [36,37]. Interestingly, in the study from Beavan et al. [36], GBA mutation carriers showed a significant deterioration in RBDQ over the 2 years of follow up, suggesting a progression of RBD symptoms already before any motor signs appear [36]. Finally, RBD prevalence significantly increased from the de-novo Parkinson's disease state to 2-year follow-up, suggesting that RBD may also represent a marker of Parkinson's disease progression [38"',39"].

\section{Conversion to synucleinopathy}

A large proportion of patients with idiopathic RBD (iRBD) are susceptible to develop a synucleinopathy $[2,40,41]$. Patients with iRBD frequently reported NMS commonly found in advanced Parkinson's disease such as dribbling saliva, cognitive deficits, and hyposmia [42]. Moreover, the presence of RBD in the prodromal Parkinson's disease phase was associated with more cognitive deficits and with greater annual rate of cognitive decline, suggesting that RBD may be a risk factor for the development of dementia in Parkinson's disease [33"]. Recent efforts aimed at identifying factors that coexist with iRBD and can predict this phenoconversion. Among 89 patients with iRBD, about half developed a synucleinopathy over 10 years. Advanced age, loss of olfaction, abnormal color vision, motor function, and nonuse of antidepressants were associated with this conversion. Combining several of these markers, subpopulations can be identified with up 
to $65 \%$ conversion rates risk in 3 years [ $\left.26^{\mathbf{*}}\right]$. Interestingly, the presence of RBD symptoms in combination with hyposmia and cognitive decline in women could effectively differentiate de-novo Parkinson's disease cases from healthy controls [43]. Pont-Sunyer et al. [10"'] assessed the possible clustering of NMS and found no associations with motor phenotypes. However dream enacting behavior was associated with frequent nightmares and constipation across all premotor timespans [10"']. Finally, EEG slowing in cortical regions during wakefulness [44"], early biochemical changes, such as the downregulation of serum for specific miRNAs (miR-19b) [45] and specific genetic polymorphisms [46] have been also suggested as promising markers to predict phenoconversion of iRBD into a synucleinopathy.

\section{PERIODIC LIMB MOVEMENTS IN SLEEP- RESTLESS LEGS SYNDROME}

In patients with Parkinson's disease, periodic limb movements in sleep (PLMS) are present in 30-80\% $[47,48]$. Only one study assessed PLMS in early Parkinson's disease and reported no differences in the PLM-Index between untreated patients with Parkinson's disease and healthy controls [14]. The prevalence of restless legs syndrome (RLS), systematically reviewed in 18 Parkinson's disease cohorts by Rijsman et al. [49] varies between 0 and 50\%, although rates close to zero were reported solely in the Asian population. For RLS, most of the studies showed no increased frequency before the onset of Parkinson's disease motor signs [50,51], despite other findings in some earlier reports [52].

Recent studies support the concept of a relatively modest role of RLS as prodromal feature in Parkinson's disease. The frequency of RLS, assessed by specific questionnaires, did not differ among patients with Parkinson's disease, individuals at high risk for Parkinson's disease, and controls [53]. RLS assessed with the RLS Diagnostic Index was present at baseline in only $4.6 \%$ of newly diagnosed drug-naïve patients with Parkinson's disease and increased to $6.5 \%$ after 2 years and $16.3 \%$ after 4 years, suggesting that, in most patients, RLS is a complication rather than a prodromal feature of Parkinson's disease [54"]. In the same study, RLS severity, assessed with the IRLSSG rating scale, was not related to Parkinson's disease progression [54"].

In line with these findings, the presence of RLS was not associated with risk for Parkinson's disease [52]. Notably, the presence of RLS in the premotor Parkinson's disease phase was related with delayed Parkinson's disease onset, reduced dyskinesia occurrence, and possibly slower progression of Parkinson's disease in comparison to matched patients with Parkinson's disease without RLS [55]. Genetic data strengthen further the notion that RLS and Parkinson's disease are likely to be distinct entities. Gan-Or et al. [56] analyzed the genetic profile of patients with Parkinson's disease and healthy controls regarding known and well-validated genetic RLS risk markers, and found no association with increased Parkinson's disease risk [56].

\section{SLEEP DISORDERED BREATHING}

The prevalence of sleep disordered breathing (SDB) in Parkinson's disease varies between 20 and 66\% $[57,58]$, claiming higher rates than in the general population $(9-17 \%$ among 50 - to 70 -year-old individuals [59]) and an association with disease progression $[60,61]$. However, other studies reported a similar or even reduced prevalence of SDB in Parkinson's disease compared to healthy controls $[62,63]$. This discrepancy might reflect the small sample sizes, differences in methodology of the assessments and bias in patients selection and therefore the data should be interpreted with caution.

Although there are polysomnographic studies reporting the Apnea-Hypopnea-Index (AHI), to our best knowledge, studies assessing the prevalence of obstructive sleep apnea (OSA) in premotor or drug-naïve early motor Parkinson's disease are lacking. Recently, two population-based studies from Taiwan assessed the risk for developing Parkinson's disease in patients with OSA $[64,65]$. The incidence of Parkinson's disease in the OSA cohort was approximately two times higher than that in individuals without OSA with women aged 50-69 and individuals with insomnia being at high risk [64]. These findings were confirmed by Sheu et al. [65].

\section{EXCESSIVE DAYTIME SLEEPINESS}

Excessive daytime sleepiness (EDS) affects 15-50\% of patients with Parkinson's disease [66]. With disease progression, EDS becomes very frequent and prominent [67], and the addition of dopaminergic medication appears to worsen its severity [66]. In early studies, subjective sleepiness and short sleep latencies in the multiple sleep latency test indicative of objective EDS were present in some drug-naïve patients with Parkinson's disease but not more often than in controls [68-70]. However, men with EDS had a three-fold higher risk of developing Parkinson's disease than controls without EDS [71].

Recently, studies based solely on subjective tools (e.g. ESS) to assess EDS in the early Parkinson's disease phase reported controversial results. Two 
studies reported a two-fold prevalence of EDS in newly diagnosed drug-naïve patients with Parkinson's disease compared to controls $\left[10^{-\prime}, 72^{\prime \prime}\right]$. In the ONSET Parkinson's disease study, EDS was assessed as part of the overall NMS burden and was frequently perceived by patients with Parkinson's disease more than 10 years prior to the motor phase [10"']. Five years after Parkinson's disease diagnosis, EDS prevalence was three times higher compared to baseline and main risk factors for developing EDS was an increased sleepiness score and the use of dopamine agonists at baseline [72"]. Mollenhauer et al. [38"'] confirmed the findings regarding the progressive worsening of EDS over the course of Parkinson's disease. However, at baseline the prevalence of EDS did not differ between drug-naïve patients with Parkinson's disease and controls [38"-]. Similarly, EDS did not differ between 423 untreated newly diagnosed patients with Parkinson's disease from the PPMI study and 196 matched controls [73]. Finally, Pont-Sunyer et al. [74] reported EDS to start after the onset of parkinsonism.

Demonstration that EDS might be a risk factor for neurodegeneration came recently from Arnulf et al. [75"] The authors assessed EDS with the ESS and concluded that EDS is frequent in patients with iRBD and that an ESS $>8$ at the time of iRBD diagnosis predicts more rapid conversion to parkinsonism and dementia [75"].

\section{FATIGUE}

Fatigue is present in about $60 \%$ (range between 33 and 70\%) [76,77] of individuals with Parkinson's disease. As the disease progresses, fatigue becomes very prominent and disabling [78] and has a negative impact on quality of life [79]. The addition of dopaminergic medication seems to worsen its severity. However, most of the data suggest that fatigue is related to disease progression, even in the premotor phase [80-82].

Recent studies support this notion: fatigue was the most frequently reported NMS in drug-naïve and treated patients with Parkinson's disease and its prevalence $(57 \%)$ did not differ between the two groups. Demonstration that fatigue is part of the prodromal Parkinson's disease phase came from two recent studies. Schrag et al. [11"], based on medical records of 8166 individuals with and 46755 individuals without $\mathrm{PD}$, reported that fatigue precedes for several years the onset of typical motor signs and its prevalence is higher among individuals who will develop Parkinson's disease than those who will not. In agreement, fatigue, assessed by NMS-Questionnaire, was four-fold more frequent in 109 drug-naïve patients with Parkinson's disease compared to controls and was perceived up to 10 years prior to the onset of motor signs. In the same study, fatigue in combination with other NMS increased the ability to discriminate Parkinson's disease from controls, identifying fatigue as a possible biomarker of Parkinson's disease [10"'].

\section{SLEEP-WAKE NEUROBIOLOGY IN PRODROMAL PARKINSON'S DISEASE}

In recent years, new hypotheses involving dopaminergic and nondopaminergic mechanisms emerged concerning sleep-wake neurobiology in Parkinson's disease (Table 1).

The dopaminergic system is related to the regulation of sleep and wakefulness at several levels. First, dopamine is implicated in circadian mechanisms including the light input and adaptation in the retina $[102,103]$ and the regulation of specific hormones, such as cortisol, prolactin, and melatonin [104]. The interaction between dopamine and melatonin [105], a major regulator of the circadian rhythm, seems to be closely linked to Parkinson's disease progression [106,107]. Second, the loss of dopaminergic transmission in Parkinson's disease not only affects the nigro-striatal projection but also dopaminergic circuits between basal ganglia and brainstem structures (particularly the pedunculopontine and laterodorsal tegmental nucleus), where important neurons of the arousal pathway and for the modulation of sleep stages are located $[1,101,108]$. Finally, mesocortical dopamine neurons innervate limbic areas (thalamus and hypothalamus) $[1,101]$, where important regulators of sleep-wake cycle are located: a) the master circadian clock in the suprachiasmatic nuclei of the hypothalamus, which mainly involves coordinated expression and activation of various clock genes [109-114] and regulation of melatonin production [115], and b) orexin/hypocretin-containing neurons, important excitatory neuromodulators of sleep homeostasis [116], which interestingly seem to be progressively reduced over the course of Parkinson's disease [117].

The degeneration of other, non dopaminergic sleep-wake networks in the brainstem is likely to play also a crucial role in SWD in Parkinson's disease. Lower brainstem nuclei including the cholinergic pedunculopontine nucleus (PPN), the noradrenergic locus coeruleus, and the serotonergic raphe nuclei are directly involved with the regulation of both wakefulness and sleep [108]. It has been suggested, in agreement with the proposed Lewy pathology staging scheme [118], that SWD in the prodromal phase of Parkinson's disease are directly 


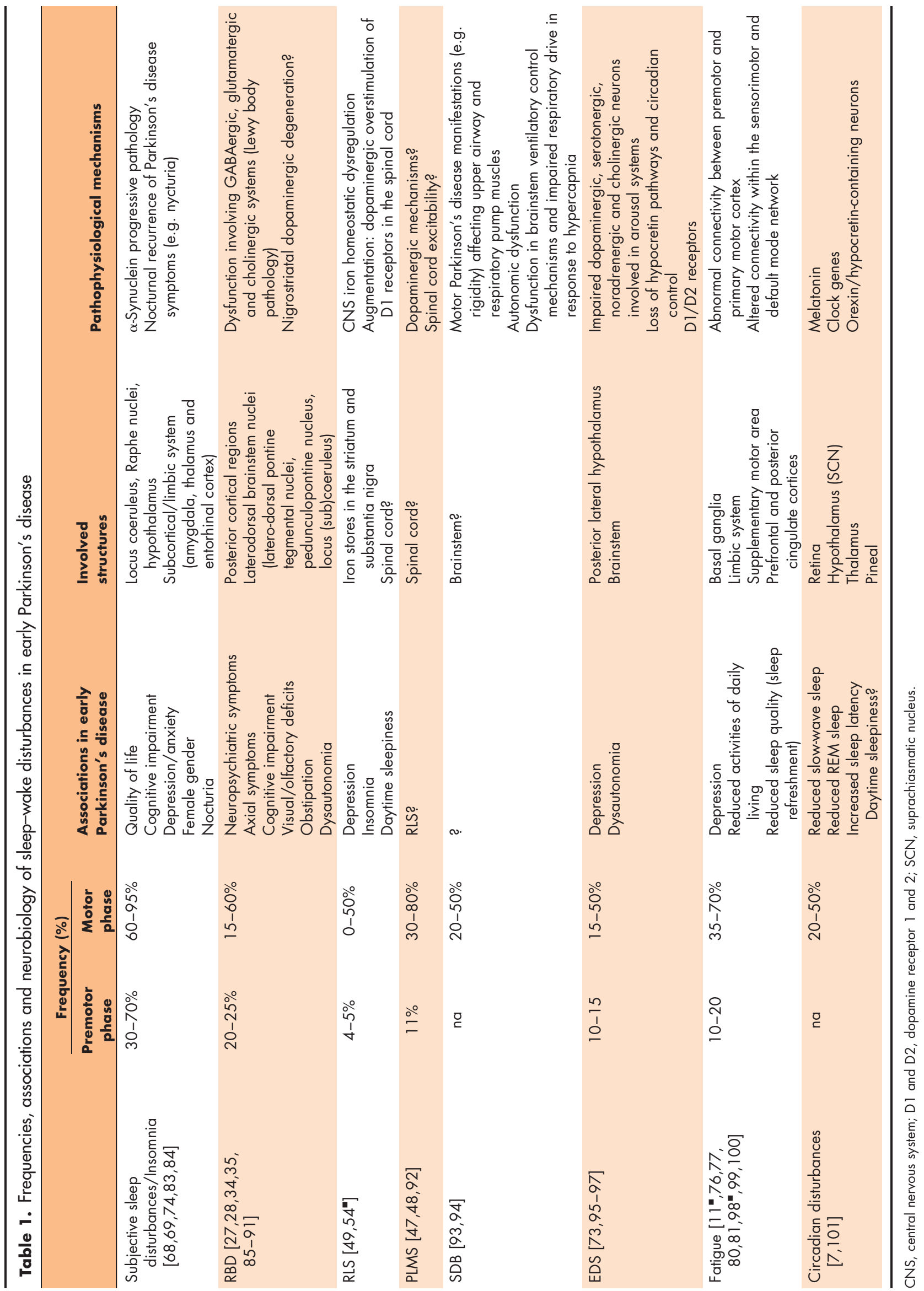


related to neurodegeneration in these brainstem nuclei $[2,3,84,119]$. Recently, De Natale et al. [120] showed that abnormalities in brainstem reflexes (vestibular-evoked myogenic potential) were more severe in patients with advanced Parkinson's disease compared with patients in the early phase and were significantly correlated with high scores on RBD Screening Questionnaire, an indicator for RBD severity. Similarly, dysfunction in brainstem ventilatory control mechanisms may be the link for the involvement of Parkinson's disease in the pathogenesis of SDB [93,94]. In contrast, Qamhawi et al. [121"] using ${ }^{123}$ I-FP-CIT single photon emission computed tomography showed that tremor but not SWD, including fatigue, EDS, and RBD is associated with dopamine and serotonin transporter availability in raphe nuclei in early Parkinson's disease.

Apart from the brainstem, other brain regions involving nondopaminergic pathways have been linked to SWD in early Parkinson's disease. Recently, Tessitore et al. [98"] using resting-state functional MRI showed that patients with Parkinson's disease and fatigue exhibited altered connectivity in the supplementary motor area as well as in the prefrontal and posterior cingulate cortices, suggesting alterations in the sensorimotor and the default mode network respectively. Wen et al. [122"] confirmed previous reports that EDS in drug-naïve patients with Parkinson's disease is related to altered neural activity in fronto-temporal and limbic areas, probably reflecting a dysfunction of the arousal system. Similarly, the slow-to-fast power ratio in restingstate waking EEG was significantly higher in frontal, central, parietal, temporal, and occipital regions in patients with iRBD who developed a synucleinopathy compared to those who did not [44"]. Finally, the presence of RBD in drug-naïve de-novo patients with Parkinson's disease was associated with brain glucose hypometabolism in posterior cortical regions [123"].

\section{CONCLUSION}

SWD are prevalent in Parkinson's disease. Although the frequency and often the severity of SWD increase with advancing Parkinson's disease, they occur in all stages of the disease. An increasing number of observational studies provide evidence regarding the prevalence of SWD in the prodromal phase and supported their role as risk factors but probably also as driving force of year-long degenerative processes that leads to the full Parkinson's disease phenotype. As our understanding of sleep-wake neurobiology increases, several hypotheses are emerging concerning the pathophysiology of SWD in premotor and early Parkinson's disease.
Taking into account the knowledge on SWD when considering the diagnosis of Parkinson's disease may facilitate the identification of individuals at high risk of developing Parkinson's disease. Future disease-modifying therapies will probably be at their most effective in patients in the earliest stage of the disease, before neurodegeneration leads to significant neuronal loss.

\section{Acknowledgements}

We would like to thank Alexios Bargiotas for his assistance in developing the graphics of the study.

\section{Financial support and sponsorship}

None.

\section{Conflicts of interest}

Panagiotis Bargiotas is currently receiving a grant from the University Hospital Bern, Inselspital (Insel-Grant), Bern, Switzerland.

Michael Schuepbach reports receiving consulting fees from Medtronic, Boston Scientific and Aleva, and lecture honoraria from Zambon, Merz Pharma, as well as grant support from Medtronic, Boston Scientific, Ipsen, Actelion. Furthermore, he received congress fees and travel reimbursements from Boston Scientific and Zambon. Claudio Bassetti has received honoraria for consultancy, lectures, and board memberships from the following institutions/companies: Jazz, Servier, UCB, Zambon. His research is currently supported by grants of the following institutions/companies: Swiss National Science Foundation (SNF), ResMed, Respironics, Vifor Pharma, UCB Pharma, Schweizerische Herzstiftung, Tropos Stiftung, Parkinson Schweiz.

\section{REFERENCES AND RECOMMENDED \\ READING}

Papers of particular interest, published within the annual period of review, have been highlighted as:

- of special interest

-1. of outstanding interest

1. Videnovic A, Golombek D. Circadian and sleep disorders in Parkinson's disease. Exp Neurol 2013; 243:45-56.

2. Iranzo A, Molinuevo JL, Santamaria J, et al. Rapid-eye-movement sleep behaviour disorder as an early marker for a neurodegenerative disorder: a descriptive study. Lancet Neurol 2006; 5:572-577.

3. Boeve BF, Dickson DW, Olson EJ, et al. Insights into REM sleep behavior disorder pathophysiology in brainstem-predominant Lewy body disease. Sleep Med 2007; 8:60-64.

4. Claassen DO, Josephs KA, Ahlskog JE, et al. REM sleep behavior disorder preceding other aspects of synucleinopathies by up to half a century. Neurology 2010; 75:494-499.

5. Barone $\mathrm{P}$, Antonini $\mathrm{A}$, Colosimo $\mathrm{C}$, et al. The PRIAMO study: a multicenter assessment of nonmotor symptoms and their impact on quality of life in Parkinson's disease. Mov Disord 2009; 24:1641-1649.

6. Stefansdottir S, Gjerstad MD, Tysnes OB, Larsen JP. Subjective sleep problems in patients with early Parkinson's disease. Eur J Neurol 2012; 19:1575-1581.

7. Breen DP, Vuono R, Nawarathna $U$, et al. Sleep and circadian rhythm regulation in early Parkinson disease. JAMA Neurol 2014; 71:589-595.

8. Lee HM, Lee SS, Kim M, et al. Clinical phenotype of drug-naive Parkinson's disease based on nonmotor symptoms. Arch Gerontol Geriatr 2015; $61: 517-522$. 
9. Baig $F$, Lawton $M$, Rolinski $M$, et al. Delineating nonmotor symptoms in early

- Parkinson's disease and first-degree relatives. Mov Disord 2015; 30:17591766.

In this study authors investigate several NMS in the context of early Parkinson's disease. Sleep-related disturbances are frequent in the early stages of Parkinson's disease and their frequencies increase with disease progression. The study shows also the different sleep-wake profile of several Parkinson's disease phenotypes. 10. Pont-Sunyer C, Hotter A, Gaig C, et al. The onset of nonmotor symptoms in n. Parkinson's disease (the ONSET PD study). Mov Disord 2015; 30:229237.

In the present study, authors assess the prevalence and the time of onset of a variety of NMS in a group of newly diagnosed, untreated patients with Parkinson's disease and a group of age-matched and gender-matched control subjects. The study also explores possible clusters of NMS and their association with the predominant motor phenotype.

11. Schrag A, Horsfall $L$, Walters $K$, et al. Prediagnostic presentations of

- Parkinson's disease in primary care: a case-control study. Lancet Neurol $2015 ; 14: 57-64$.

This is a large population-based study examining the association between several prediagnostic features, including RBD, and a subsequent diagnosis of Parkinson's disease. Individuals later diagnosed with Parkinson's disease had, years before Parkinson's disease diagnosis, a higher incidence of RBD and insomnia.

12. Diederich NJ, Vaillant M, Mancuso G, et al. Progressive sleep 'destructuring' in Parkinson's disease. A polysomnographic study in 46 patients. Sleep Med 2005; 6:313-318.

13. Sixel-Doring F, Trautmann E, Mollenhauer B, Trenkwalder C. Rapid eye movement sleep behavioral events: a new marker for neurodegeneration in early Parkinson disease? Sleep 2014; 37:431-438.

14. Wetter TC, Brunner $\mathrm{H}$, Hogl $\mathrm{B}$, et al. Increased alpha activity in REM sleep in de novo patients with Parkinson's disease. Mov Disord 2001; 16:928-933.

15. Brunner $\mathrm{H}$, Wetter TC, Hogl $B$, et al. Microstructure of the nonrapid eye movement sleep electroencephalogram in patients with newly diagnosed Parkinson's disease: effects of dopaminergic treatment. Mov Disord 2002; 17:928-933.

16. Gagnon JF, Fantini ML, Bedard MA, et al. Association between waking EEG slowing and REM sleep behavior disorder in PD without dementia. Neurology $2004 ; 62: 401-406$

17. Margis R, Schonwald SV, Carvalho DZ, et al. NREM sleep alpha and sigma

- activity in Parkinson's disease: evidence for conflicting electrophysiological activity? Clin Neurophysiol 2015; 126:951-958.

This is a sleep EEG study of sleep microstructure in newly diagnosed drug-naïve patients with Parkinson's disease. Authors reported increased scalp EEG alpha and sigma activity during non-REM sleep compared to healthy controls.

18. Dang-Vu TT, McKinney SM, Buxton OM, et al. Spontaneous brain rhythms predict sleep stability in the face of noise. Curr Biol 2010; 20:R626-R627.

19. Doucette MR, Kurth S, Chevalier N, et al. Topography of slow sigma power during sleep is associated with processing speed in preschool children. Brain Sci 2015; 5:494-508.

20. Chatburn $A$, Coussens $S$, Lushington $K$, et al. Sleep spindle activity and cognitive performance in healthy children. Sleep 2013; 36:237-243.

21. Schabus $M$, Gruber $G$, Parapatics $S$, et al. Sleep spindles and their significance for declarative memory consolidation. Sleep 2004; 27:1479-1485.

22. Christensen JA, Kempfner J, Zoetmulder M, et al. Decreased sleep spindle density in patients with idiopathic REM sleep behavior disorder and patients with Parkinson's disease. Clin Neurophysiol 2014; 125:512-519.

23. Joy $S P$, Sinha $S, P a l P K$, et al. Serial macro-architectural alterations with levodopa in Parkinson's disease: Polysomnography (PSG)-based analysis. Ann Indian Acad Neurol 2015; 18:309-313.

24. Videnovic A, Willis GL. Circadian system - a novel diagnostic and ther-

- apeutic target in Parkinson's disease? Mov Disord 2016; 31:260-269.

This article is a thorough review of the existing circadian research in Parkinson's disease.

25. Lauretti E, Di Meco A, Merali S, Pratico D. Circadian rhythm dysfunction: a novel environmental risk factor for Parkinson's disease. Mol Psychiatry 2016. [Epub ahead of print]

26. Postuma RB, Gagnon JF, Bertrand JA, et al. Parkinson risk in idiopathic REM

- sleep behavior disorder: preparing for neuroprotective trials. Neurology 2015; 84:1104-1113.

This prospective 10-year follow-up cohort highlights the predictive value of several markers for the development of a synucleinopathy in patients with idiopathic RBD and explores the true risk for this phenoconversion.

27. Gagnon JF, Bedard MA, Fantini ML, et al. REM sleep behavior disorder and REM sleep without atonia in Parkinson's disease. Neurology 2002; 59:585589.

28. Poryazova R, Oberholzer M, Baumann CR, Bassetti CL. REM sleep behavior disorder in Parkinson's disease: a questionnaire-based survey. J Clin Sleep Med 2013; 9:55-59.

29. Sixel-Doring F, Trautmann E, Mollenhauer B, Trenkwalder C. Associated factors for REM sleep behavior disorder in Parkinson disease. Neurology 2011; 77:1048-1054.

30. Gjerstad MD, Boeve B, Wentzel-Larsen T, et al. Occurrence and clinical correlates of REM sleep behaviour disorder in patients with Parkinson's disease over time. J Neurol Neurosurg Psychiatry 2008; 79:387-391.
31. Rolinski M, Szewczyk-Krolikowski K, Tomlinson PR, et al. REM sleep behaviour disorder is associated with worse quality of life and other nonmotor features in early Parkinson's disease. J Neurol Neurosurg Psychiatry 2014; 85:560-566.

32. Postuma RB, Lang $A E$, Gagnon JF, et al. How does parkinsonism start? Prodromal parkinsonism motor changes in idiopathic REM sleep behaviour disorder. Brain 2012; 135 (Pt 6):1860-1870.

33. Chahine LM, Xie SX, Simuni T, et al. Longitudinal changes in cognition in

- early Parkinson's disease patients with REM sleep behavior disorder. Parkinsonism Relat Disord 2016; 27:102-106.

This prospective cohort study explores associations between RBD and cognition in patients with Parkinson's disease. Authors show that newly drug-naïve patients with Parkinson's disease with RBD have, already at baseline, more cognitive deficits and with disease progression higher rates of cognitive decline compared to patients with Parkinson's disease without RBD. These findings highlight the important prognostic value of RBD in Parkinson's disease.

34. Swallow DM, Lawton MA, Grosset KA, et al. Variation in recent onset Parkinson's disease: implications for prodromal detection. J Parkinsons Dis 2016; 6:289-300.

35. Pagonabarraga J, Martinez-Horta S, Fernandez de Bobadilla R, et al. Minor hallucinations occur in drug-naive Parkinson's disease patients, even from the premotor phase. Mov Disord 2016; 31:45-52.

36. Beavan M, McNeill A, Proukakis $C$, et al. Evolution of prodromal clinical markers of Parkinson disease in a GBA mutation-positive cohort. JAMA Neurol 2015; 72:201-208.

37. Bergareche A, Rodriguez-Oroz MC, Estanga A, et al. DAT imaging and clinical biomarkers in relatives at genetic risk for LRRK2 R1441 G Parkinson's disease. Mov Disord 2016; 31:335-343.

38. Mollenhauer B, Zimmermann J, Sixel-Doring F, et al. Monitoring of 30 marker

m. candidates in early Parkinson disease as progression markers. Neurology $2016 ; 87: 168-177$.

This longitudinal 2-year follow-up study investigates the progression of several candidate biomarkers over the course of Parkinson's disease. RBD may be a useful marker to monitor progression of Parkinson's disease. Daytime sleepiness progressively worsens over the course of Parkinson's disease.

39. Sixel-Doring F, Zimmermann J, Wegener A, et al. The evolution of REM sleep

- behavior disorder in early Parkinson disease. Sleep 2016. [Epub ahead of print]

This observational study confirms the progressive nature of RBD in early Parkinson's disease despite initiation of dopaminergic treatment. Compared to healthy controls, initially de-novo Parkinson's disease patients show a significant increase in the frequency of polysomnographic confirmed RBD in the 2-years follow-up.

40. Iranzo A, Tolosa E, Gelpi E, et al. Neurodegenerative disease status and postmortem pathology in idiopathic rapid-eye-movement sleep behaviour disorder: an observational cohort study. Lancet Neurol 2013; 12:443-453.

41. Postuma RB, Gagnon JF, Vendette M, et al. Quantifying the risk of neurodegenerative disease in idiopathic REM sleep behavior disorder. Neurology 2009; 72:1296-1300.

42. Mahlknecht $P$, Seppi $K$, Frauscher $B$, et al. Probable RBD and association with neurodegenerative disease markers: a population-based study. Mov Disord 2015; 30:1417-1421

43. Liu R, Umbach DM, Peddada SD, et al. Potential sex differences in nonmotor symptoms in early drug-naive Parkinson disease. Neurology 2015; 84:2107-2115.

44. Rodrigues Brazete J, Gagnon JF, Postuma RB, et al. Electroencephalogram

- slowing predicts neurodegeneration in rapid eye movement sleep behavior disorder. Neurobiol Aging 2016; 37:74-81.

This study examines the relationship between baseline EEG characteristics and the risk for developing a synucleinopathy in patients with iRBD. Individuals with iRBD who developed a synucleinopathy compared to those who did not (mean follow-up: 3.5 years) showed EEG slowing characterized by higher absolute delta and theta power in all cortical regions.

45. Fernandez-Santiago R, Iranzo A, Gaig C, et al. MicroRNA association with synucleinopathy conversion in rapid eye movement behavior disorder. Ann Neurol 2015; 77:895-901.

46. Gan-Or Z, Girard SL, Noreau A, et al. Parkinson's disease genetic loci in rapid eye movement sleep behavior disorder. J Mol Neurosci 2015; 56:617622.

47. Bliwise DL, Trotti LM, Yesavage JA, Rye DB. Periodic leg movements in sleep in elderly patients with Parkinsonism and Alzheimer's disease. Eur J Neurol 2012; 19:918-923

48. Poewe W, Hogl B. Akathisia, restless legs and periodic limb movements in sleep in Parkinson's disease. Neurology 2004; 63 (8 Suppl 3):S12-S16.

49. Rijsman RM, Schoolderman LF, Rundervoort RS, Louter M. Restless legs syndrome in Parkinson's disease. Parkinsonism Relat Disord 2014; 20 (Suppl 1):S5-S9.

50. Gjerstad MD, Tysnes OB, Larsen JP. Increased risk of leg motor restlessness but not RLS in early Parkinson disease. Neurology 2011; 77:1941-1946.

51. Angelini M, Negrotti $A$, Marchesi $E$, et al. A study of the prevalence of restless legs syndrome in previously untreated Parkinson's disease patients: absence of co-morbid association. J Neurol Sci 2011; 310 (1-2):286-288.

52. Wong JC, Li Y, Schwarzschild MA, et al. Restless legs syndrome: an early clinical feature of Parkinson disease in men. Sleep 2014; 37:369-372. 
53. Louter M, Maetzler W, Prinzen J, et al. Accelerometer-based quantitative analysis of axial nocturnal movements differentiates patients with Parkinson's disease, but not high-risk individuals, from controls. J Neurol Neurosurg Psychiatry 2015; 86:32-37.

54. Moccia M, Erro R, Picillo $M$, et al. A four-year longitudinal study on restless - legs syndrome in Parkinson disease. Sleep 2016; 39:405-412.

This longitudinal study explores the incidence of RLS at the time of Parkinson's disease diagnosis, and over the course of Parkinson's disease. RLS prevalence is at the time of Parkinson's disease diagnosis, similar to that described for idiopathic RLS in the general population but significantly higher in more advanced stages of Parkinson's disease.

55. Ylikoski A, Martikainen $K$, Partinen M. Parkinson's disease and restless legs syndrome. Eur Neurol 2015; $73(3-4): 212-219$.

56. Gan-Or Z, Alcalay RN, Bar-Shira A, et al. Genetic markers of restless legs syndrome in Parkinson disease. Parkinsonism Relat Disord 2015; 21:582585.

57. Alatriste-Booth V, Rodriguez-Violante M, Camacho-Ordonez A, CervantesArriaga A. Prevalence and correlates of sleep disorders in Parkinson's disease: a polysomnographic study. Arq Neuropsiquiatr 2015; 73:241245.

58. da Silva-Junior FP Jr, do Prado GF, Barbosa ER, et al. Sleep disordered breathing in Parkinson's disease: a critical appraisal. Sleep Med Rev 2014; 18:173-178.

59. Peppard PE, Young $\mathrm{T}$, Barnet $\mathrm{JH}$, et al. Increased prevalence of sleepdisordered breathing in adults. Am J Epidemiol 2013; 177:1006-1014.

60. Maria B, Sophia S, Michalis M, et al. Sleep breathing disorders in patients with idiopathic Parkinson's disease. Respir Med 2003; 97:1151-1157.

61. Cochen De Cock V, Abouda M, Leu S, et al. Is obstructive sleep apnea a problem in Parkinson's disease? Sleep Med 2010; 11:247-252.

62. Trotti LM, Bliwise DL. No increased risk of obstructive sleep apnea in Parkinson's disease. Mov Disord 2010; 25:2246-2249.

63. Zeng J, Wei M, Li T, et al. Risk of obstructive sleep apnea in Parkinson's disease: a meta-analysis. PLoS One 2013; 8:e82091.

64. Chen JC, Tsai TY, Li CY, Hwang JH. Obstructive sleep apnea and risk of Parkinson's disease: a population-based cohort study. J Sleep Res 2015; 24:432-437

65. Sheu JJ, Lee HC, Lin HC, et al. A 5-year follow-up study on the relationship between obstructive sleep apnea and Parkinson disease. J Clin Sleep Med 2015; 11:1403-1408.

66. Ondo WG, Dat Vuong K, Khan H, et al. Daytime sleepiness and other sleep disorders in Parkinson's disease. Neurology 2001; 57:1392-1396.

67. Zhu K, van Hilten JJ, Marinus J. Course and risk factors for excessive daytime sleepiness in Parkinson's disease. Parkinsonism Relat Disord 2016; 24:3440.

68. Kaynak D, Kiziltan G, Kaynak H, et al. Sleep and sleepiness in patients with Parkinson's disease before and after dopaminergic treatment. Eur J Neurol 2005; 12:199-207.

69. Buskova J, Klempir J, Majerova V, et al. Sleep disturbances in untreated Parkinson's disease. J Neurol 2011; 258:2254-2259.

70. Poryazova R, Benninger D, Waldvogel D, Bassetti CL. Excessive daytime sleepiness in Parkinson's disease: characteristics and determinants. Eur Neurol 2010; 63:129-135.

71. Abbott RD, Ross GW, White LR, et al. Excessive daytime sleepiness and subsequent development of Parkinson disease. Neurology 2005; 65:14421446 .

72. Tholfsen LK, Larsen JP, Schulz J, et al. Development of excessive daytime

- sleepiness in early Parkinson disease. Neurology 2015; 85:162-168.

This longitudinal study explores the incidence of EDS at the time of Parkinson's disease diagnosis and over the course of the disease. EDS prevalence is similar to that described for EDS in the general population at the time of Parkinson's disease diagnosis, but significantly higher in more advanced stages of Parkinson's disease. Risk and associated factors for this increase are examined.

73. Simuni $T$, Caspell-Garcia C, Coffey $C$, et al. Correlates of excessive daytime sleepiness in de novo Parkinson's disease: a case control study. Mov Disord 2015; 30:1371-1381.

74. Pont-Sunyer C, Iranzo A, Gaig C, et al. Sleep disorders in parkinsonian and nonparkinsonian LRRK2 mutation carriers. PLoS One 2015; 10:e0132368.

75. Arnulf I, Neutel D, Herlin B, et al. Sleepiness in idiopathic REM sleep behavior

- disorder and Parkinson disease. Sleep 2015; 38:1529-1535.

This study explores sleepiness (measured by ESS) in the context of idiopathic RBD. Patients with idiopathic RBD are sleepier than controls and high ESS scores at time of RBD diagnosis predicts more rapid conversion of idiopathic RBD to parkinsonism and dementia.

76. Friedman $\mathrm{JH}$, Brown RG, Comella $\mathrm{C}$, et al. Fatigue in Parkinson's disease: a review. Mov Disord 2007; 22:297-308.

77. Friedman JH, Abrantes A, Sweet LH. Fatigue in Parkinson's disease. Expert Opin Pharmacother 2011; 12:1999-2007.

78. Kluger BM, Herlofson $\mathrm{K}$, Chou KL, et al. Parkinson's disease-related fatigue: a case definition and recommendations for clinical research. Mov Disord 2016; 31:625-631.

79. Friedman $\mathrm{JH}$, Alves $\mathrm{G}$, Hagell $\mathrm{P}$, et al. Fatigue rating scales critique and recommendations by the Movement Disorders Society task force on rating scales for Parkinson's disease. Mov Disord 2010; 25:805-822.
80. Schifitto $\mathrm{G}$, Friedman $\mathrm{JH}$, Oakes $\mathrm{D}$, et al. Fatigue in levodopa-naive subjects with Parkinson disease. Neurology 2008; 71:481-485.

81. Kang SY, Ma HI, Lim YM, et al. Fatigue in drug-naive Parkinson's disease. Eur Neurol 2013; $70(1-2): 59-64$.

82. Herlofson K, Ongre SO, Enger LK, et al. Fatigue in early Parkinson's disease. Minor inconvenience or major distress? Eur J Neurol 2012; 19:963-968.

83. Gjerstad MD, Wentzel-Larsen T, Aarsland D, Larsen JP. Insomnia in Parkinson's disease: frequency and progression over time. J Neurol Neurosurg Psychiatry 2007; 78:476-479.

84. Kalaitzakis ME, Gentleman SM, Pearce RK. Disturbed sleep in Parkinson's disease: anatomical and pathological correlates. Neuropathol Appl Neurobiol 2013; 39:644-653

85. Grinberg LT, Rueb U, Alho AT, Heinsen H. Brainstem pathology and nonmotor symptoms in PD. J Neurol Sci 2010; $289(1-2): 81-88$.

86. Luppi $\mathrm{PH}$, Clement $\mathrm{O}$, Valencia Garcia $\mathrm{S}$, et al. New aspects in the pathophysiology of rapid eye movement sleep behavior disorder: the potential role of glutamate, gamma-aminobutyric acid, and glycine. Sleep Med 2013; 14:714-718.

87. Kim YK, Yoon IY, Kim JM, et al. The implication of nigrostriatal dopaminergic degeneration in the pathogenesis of REM sleep behavior disorder. Eur J Neurol 2010; 17:487-492.

88. Iranzo A. Parkinson disease and sleep: sleep-wake changes in the premotor stage of Parkinson disease; impaired olfaction and other prodromal features. Curr Neurol Neurosci Rep 2013; 13:373.

89. Postuma RB, Lang AE, Massicotte-Marquez J, Montplaisir J. Potential early markers of Parkinson disease in idiopathic REM sleep behavior disorder. Neurology 2006; 66:845-851.

90. Iwanami M, Miyamoto $T$, Miyamoto $M$, et al. Relevance of substantia nigra hyperechogenicity and reduced odor identification in idiopathic REM sleep behavior disorder. Sleep Med 2010; 11:361-365.

91. Iranzo A, Santamaria J, Tolosa E. The clinical and pathophysiological relevance of REM sleep behavior disorder in neurodegenerative diseases. Sleep Med Rev 2009; 13:385-401.

92. Bara-Jimenez W, Aksu M, Graham B, et al. Periodic limb movements in sleep: state-dependent excitability of the spinal flexor reflex. Neurology 2000; 54:1609-1616.

93. Seccombe LM, Giddings HL, Rogers PG, et al. Abnormal ventilatory control in Parkinson's disease - further evidence for nonmotor dysfunction. Respir Physiol Neurobiol 2011; 179 (2-3):300-304.

94. Kaminska M, Lafontaine AL, Kimoff RJ. The interaction between obstructive sleep apnea and Parkinson's disease: possible mechanisms and implications for cognitive function. Parkinsons Dis 2015; 2015:849472.

95. Rye DB. The two faces of Eve: dopamine's modulation of wakefulness and sleep. Neurology 2004; 63 (8 Suppl 3):S2-S7.

96. Suzuki K, Miyamoto M, Miyamoto T, et al. Sleep disturbances associated with Parkinson's disease. Parkinsons Dis 2011; 2011:219056.

97. Arnulf I. Excessive daytime sleepiness in parkinsonism. Sleep Med Rev 2005; 9:185-200.

98. Tessitore A, Giordano A, De Micco R, et al. Functional connectivity under-

- pinnings of fatigue in 'Drug-Naive' patients with Parkinson's disease. Mov Disord 2016. [Epub ahead of print]

This is a resting-state functional MRI imaging study of the neural basis of fatigue in drug-naive patients with Parkinson's disease. Its novel contribution to the field is that it shows that fatigue is associated with brain functional abnormalities in the sensorimotor and default mode network, implicating nondopaminergic pathways.

99. Pavese N, Metta V, Bose SK, et al. Fatigue in Parkinson's disease is linked to striatal and limbic serotonergic dysfunction. Brain 2010; 133:3434-3443.

100. Lou JS, Benice T, Kearns G, et al. Levodopa normalizes exercise related cortico-motoneuron excitability abnormalities in Parkinson's disease. Clin Neurophysiol 2003; 114:930-937.

101. Monti JM, Monti D. The involvement of dopamine in the modulation of sleep and waking. Sleep Med Rev 2007; 11:113-133.

102. Dorenbos $\mathrm{R}$, Contini $\mathrm{M}$, Hirasawa $\mathrm{H}$, et al. Expression of circadian clock genes in retinal dopaminergic cells. Vis Neurosci 2007; 24:573-580.

103. Witkovsky P. Dopamine and retinal function. Doc Ophthalmol 2004; 108:17-40

104. Barcia C, Bautista V, Sanchez-Bahillo A, et al. Circadian determinations of cortisol, prolactin and melatonin in chronic methyl-phenyl-tetrahydropyridinetreated monkeys. Neuroendocrinology 2003; 78:118-128.

105. Cahill GM, Grace MS, Besharse JC. Rhythmic regulation of retinal melatonin: metabolic pathways, neurochemical mechanisms, and the ocular circadian clock. Cell Mol Neurobiol 1991; 11:529-560.

106. Fertl E, Auff E, Doppelbauer A, Waldhauser F, et al. Circadian secretion pattern of melatonin in de novo parkinsonian patients: evidence for phaseshifting properties of I-dopa. J Neural Transm Park Dis Dement Sect 1993; 5:227-234

107. Bordet R, Devos D, Brique S, et al. Study of circadian melatonin secretion pattern at different stages of Parkinson's disease. Clin Neuropharmacol 2003; 26:65-72.

108. Saper CB, Chou TC, Scammell TE. The sleep switch: hypothalamic control of sleep and wakefulness. Trends Neurosci 2001; 24:726-731.

109. Fifel K, Vezoli J, Dzahini K, et al. Alteration of daily and circadian rhythms following dopamine depletion in MPTP-treated nonhuman primates. PLoS One 2014; 9:e86240. 
110. Abarca $C$, Albrecht $U$, Spanagel R. Cocaine sensitization and reward are under the influence of circadian genes and rhythm. Proc Natl Acad Sci U S A 2002; 99:9026-9030.

111. Baltazar RM, Coolen LM, Webb IC. Medial prefrontal cortex inactivation attenuates the diurnal rhythm in amphetamine reward. Neuroscience 2014; 258:204-210.

112. Feillet CA, Mendoza J, Albrecht $U$, et al. Forebrain oscillators ticking with different clock hands. Mol Cell Neurosci 2008; 37:209-221.

113. Uz $T$, Ahmed $R$, Akhisaroglu $M$, et al. Effect of fluoxetine and cocaine on the expression of clock genes in the mouse hippocampus and striatum. Neuroscience 2005; 134:1309-1316.

114. Gravotta L, Gavrila AM, Hood S, Amir S. Global depletion of dopamine using intracerebroventricular 6-hydroxydopamine injection disrupts normal circadian wheel-running patterns and PERIOD2 expression in the rat forebrain. $J$ Mol Neurosci 2011; 45:162-171.

115. Dibner $\mathrm{C}$, Schibler U, Albrecht U. The mammalian circadian timing system: organization and coordination of central and peripheral clocks. Annu Rev Physiol 2010; 72:517-549.

116. Sutcliffe JG, de Lecea L. The hypocretins: excitatory neuromodulatory peptides for multiple homeostatic systems, including sleep and feeding. J Neurosci Res 2000; 62:161-168.

117. Wienecke $M$, Werth $E$, Poryazova $R$, et al. Progressive dopamine and hypocretin deficiencies in Parkinson's disease: is there an impact on sleep and wakefulness? J Sleep Res 2012; 21:710-717.

118. Braak $H$, Del Tredici $K$, Rub $U$, et al. Staging of brain pathology related to sporadic Parkinson's disease. Neurobiol Aging 2003; 24: $197-211$.
119. Boeve BF, Silber MH, Saper CB, et al. Pathophysiology of REM sleep behaviour disorder and relevance to neurodegenerative disease. Brain 2007; 130 (Pt 11):2770-2788

120. de Natale ER, Ginatempo F, Paulus KS, et al. Paired neurophysiological and clinical study of the brainstem at different stages of Parkinson's Disease. Clin Neurophysiol 2015; 126:1871-1878.

121. Qamhawi $Z$, Towey $D$, Shah $B$, et al. Clinical correlates of raphe serotonergic - dysfunction in early Parkinson's disease. Brain 2015; 138 (Pt 10):29642973.

This is a large neuroimaging study examining possible association of the raphe nuclei with motor and nonmotor symptoms in early drug-naive Parkinson's disease. Authors suggest that raphe serotonin transporter availability is related to motor symptoms but not to SWD, including fatigue, EDS, and RBD.

122. Wen MC, Ng SY, Heng HS, et al. Neural substrates of excessive daytime - Sleepiness in early drug naive Parkinson's disease: A resting state functional MRI study. Parkinsonism Relat Disord 2016; 24:63-68.

This is a resting-state functional MRI imaging study of the neural basis of EDS in Parkinson's disease, reporting functional abnormalities in frontal and cerebellar areas in sleepy drug-naive patients with Parkinson's disease.

123. Arnaldi $D$, Morbelli $S$, Brugnolo $A$, et al. Functional neuroimaging and clinical - features of drug naive patients with de novo Parkinson's disease and probable RBD. Parkinsonism Relat Disord 2016. [Epub ahead of print]

This study explores the functional neuroimaging phenotype of early patients with Parkinson's disease with and without probable RBD, using ${ }^{123}$ I-FP-CIT-SPECT and ${ }^{18} \mathrm{~F}$-FDG-PET. Patients with RBD had a higher prevalence of $\mathrm{MCl}$, obstipation and olfaction and exhibited more severe nigro-striatal dopaminergic impairment and an altered brain glucose metabolism, mainly in posterior cortical regions. 